\title{
Agrarpolitik 2002: ökonomische und ökologische Auswirkungen auf die Regionen der Schweiz
}

\section{Einleitung}

Seit 1992 ist die schweizerische Agrarpolitik in einem markanten Umbau. Dieser umfaßt folgende drei Schritte:

- Abänderung des (alten) Art. 29 des Landwirtschaftsgesetzes und seine Ergänzung durch die (neuen) Artikel 31a und 31b

- Beitritt zum neuen WTO/Gatt-Vertrag (Ergebnis der Uruguay-Runde)

- Vollständiger Umbau des Landwirtschaftsgesetzes, bekannt unter der Bezeichnung Agrarpolitik 2002 (AP 2002) (zurzeit läuft die Vernehmlassung, Februar 1996)

Diese Neuorientierung der schweizerischen Agrarpolitik hat eine interne und eine externe Ursache. Die interne Ursache besteht darin, daß wegen der bis 1992 gewährten kostendeckenden Preise, des perfekten Außenhandelsschutzes und der Exportsubventionen folgende Probleme entstanden:

- kaum finanzierbare Marktüberschüsse,

- eine umweltgefährdende Produktionsintensität und

- interventionistisch bedingte Einkommensunterschiede innerhalb der Landwirtschaft (Berg-/Talgebiet, kleinere/größere Betriebe, Milch-/Fleischproduktionsbetriebe).

Die externe Ursache läßt sich mit folgenden Stichworten angeben: internationale Agrarmarktüberschüsse infolge des Agrarprotektionismus der Industrieländer; totaler Preiszusammenbruch auf den Agrarmärkten in den achtziger Jahren; zunehmende Armut in vielen Entwicklungsländern; Zusammenbruch der Planwirtschaften. Dies waren bekanntlich die Hauptgründe für die Uruguay-Runde im Rahmen des Gatt.

Nach diesen einleitenden Bemerkungen treten wir nun auf die Titelfrage dieses Beitrages ein, nämlich ob die drei einleitend genannten Gesetzesänderungen spezifisch ökonomische und auch ökologische Auswirkungen auf die Regionen der Schweiz haben bzw. noch haben werden.

\section{Ergänzung des Landwirtschaftsgesetzes} mit den Artikeln 31 a und 31 b von 1992

Mit dieser Gesetzesänderung wurde die Trennung der Produktpreis- von der Einkommenspolitik für die ganze Landwirtschaft vollzogen. Ab jenem Zeitpunkt wurde der produktbezogene Agrarschutz (Milchpreise, Getrei- depreise usw.) schrittweise gesenkt und wurden die Einkommen zunehmend über ergänzende produktionsunabhängige Direktzahlungen ausgeglichen. Weil diese Direktzahlungen produktionsunabhängig, also an Betriebe und an die Flächen gebunden sind, führte bereits dieser Umbau auch zu regional unterschiedlichen Auswirkungen. Produktionsintensive Betriebe hatten durch den Preisabbau relativ größere Verluste als extensiv produzierende, da alle die gleichen Zahlungen pro Fläche bzw. pro Betrieb erhielten. Die Absicht dieser Gesetzesänderung war, eine zwar flächendeckende, aber extensivere Landwirtschaft herbeizuführen. Abbildung 1 zeigt, wie sich seither diesbezüglich die Bundesausgaben tatsächlich von der Produktionsstützung auf Direktzahlungen verlagerten.

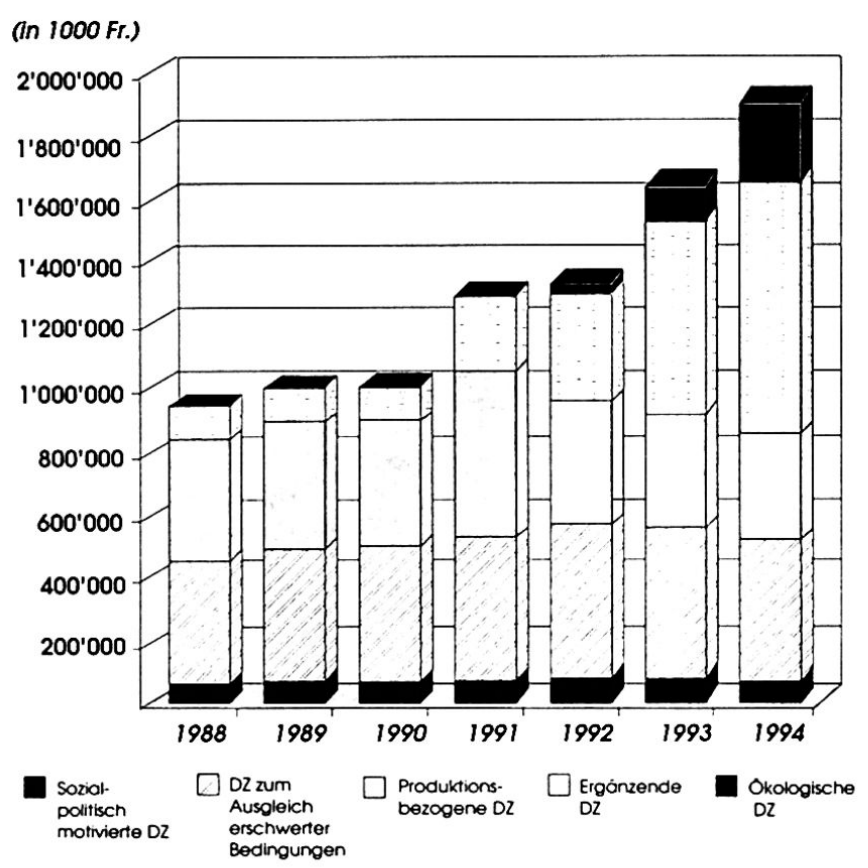

Abb.1 Umbau der Agrarstützung seit 1990.

Peter Rieder, Prof. Dr., Institut für Agranwirtschaft, ETH Zentrum, 8092 Zürich 
Tab.1 Veränderungen der Anzahl Betriebe 1990 bis 1994 in den Kantonen Bern und Graubünden.

\begin{tabular}{|l|c|l|c|}
\hline Kanton Bern & $\begin{array}{c}\text { \% Veränderung } \\
1990-1994 \text { jährlich }\end{array}$ & Kanton Graubünden & $\begin{array}{c}\text { \% Veränderung } \\
1990-1994 \text { jährlich }\end{array}$ \\
\hline Talzone & $-3,4$ & Talzone & $-5,8$ \\
\hline Voralpine Hügelzone & $-2,8$ & Voralpine Hügelzone & $-7,5$ \\
\hline Bergzone 1 & $-2,3$ & Bergzone 1 & $-5,3$ \\
\hline Bergzone 2 & $-2,3$ & Bergzone 2 & $-2,8$ \\
\hline Bergzone 3 & -2 & Bergzone 3 & $-4,5$ \\
\hline Bergzone 4 & $+3,5$ & Bergzone 4 & $+0,2$ \\
\hline
\end{tabular}

Ob die Agrarstrukturen auf diesen Umbau auch erwartungsgemäß reagiert haben, läßt sich wegen der wenigen Jahre erst durch gezielte Einzeluntersuchungen aufzeigen. Wir haben solche an den verfügbaren Daten in den Kantonen Bern und Graubünden vorgenommen. Die Ergebnisse enthält die Tabelle 1.

Aus den Angaben in Tabelle 1 lassen sich gleiche Tendenzen herauslesen. Ausgehend von einer erwarteten durchschnittlichen jährlichen Abnahme der Anzahl Betriebe von zwei Prozent stellt man fest, daß die Abnahmen in den intensiv produzierenden Talregionen größer als in den extensiver produzierenden Bergzonen sind. Diese wenn auch als provisorisch zu betrachtenden Veränderungen weisen zumindest darauf hin, daß regional unterschiedliche Tendenzen zu erwarten sind. Auch dürfte die Agrarproduktion tendenziell ökologischer werden und dürften die Agrarüberschüsse abnehmen. Bekannt ist ferner, daß relativ viele Landwirte auf die speziellen ökologischen Beiträge nach Art. $31 \mathrm{lb}$ reagiert bzw. ihre Betriebe auf diese Produktionsformen umgestellt haben (BAUR 1995).

\section{Gatt-bedingte regionale Auswirkungen}

Die wichtigsten drei agrarbezogenen Verpflichtungen der Uruguay-Runde des Gatt sind in Tabelle 2 zusammengestellt. Erstens müssen die Agrarpreise gesenkt werden, zweitens muß ein minimaler Marktzutritt für Agrarprodukte gewährt werden, und drittens sind die Exportsubventionen zu kürzen. Besonders zu beachten ist, daß die interne Stützung nur dort abgebaut werden muß, wo keine Produktionsbeschränkungen angewendet werden (Kontingentierungen). In diesen Fällen sind nach wie vor produktgebundene Ausgleichszahlungen möglich. Ferner sind (in der gleichen Kolonne aufgeführt) produktunabhängige Zahlungen nach wie vor möglich (green box).

$\mathrm{Zu}$ den Auswirkungen der Gatt-Beschlüsse wurden sehr ausfuihrliche Untersuchungen angestellt (RIEDER et al. 1995). Folgende Ergebnisse sind für die hier vorliegende Fragestellung relevant:

- Die weitere Senkung der Agrarpreise und deren teilweiser Ausgleich durch Direktzahlungen werden die

Tab. 2 Elemente der Schlußakte der Uruguay-Runde im Agrarbereich.

\begin{tabular}{|l|l|l|}
\hline Interne Stützung & Marktzutritt & Exportsubventionen \\
\hline nicht produktgebunden: & Tarifizierung: & bezüglich Menge: \\
(«green box»; z. B. Direkt- & ohne Ausnahme & Abbau um 21\% (ohne verarbeitete \\
zahlungen) & Zollabbau: 36\% im Mittel & Landwirtschaftsprodukte) \\
keine Reduktion & (mind. 15\%) & bezüglich Budget: \\
produktgebunden, & Marktzutritt: & \\
aber produktionsbeschränkend: & - Beibehaltung der Import- & \\
keine Reduktion während der & möglichkeiten von 1986-88 & \\
Übergangsperiode & zu gleichen Bedingungen \\
produktgebunden (Rest): & - Steigerung der Import- & \\
Reduktion um 20\% & möglichkeiten von 3 auf 5\% & \\
& des Konsums bis zum Ende & \\
Basisjahre: 1986-88 & der Übergangsperiode & \\
Übergangsperiode: & Basisjahre: 1986-88 & Basisjahre: 1986-90 \\
6 Jahre & Übergangsperiode: & Übergangsperiode: \\
\hline
\end{tabular}

Quelle: Bundesamt für Landwirtschaft 
bereits oben erwähnte Tendenz der relativen Bevorzugung extensiv produzierender Betriebe im Vergleich zu eher intensiven Betrieben verstärken. Konkret bedeutet dies eine relative Bevorzugung des Berggebietes und der Regionen mit eher größeren Betrieben (Westschweiz).

- Weil im Laufe der Anpassung bis zum Jahre 2002 die Produktpreise gesenkt und die Direktzahlungen ausgebaut werden sollen, interessiert die Frage des dannzumaligen Anteils der Direktzahlungen am Gesamterlös der Landwirte in verschiedenen Regionen. Nach unseren Berechnungen (RIEDER et al. 1995) schwanken diese Anteile von rund $8 \%$ im Talgebiet bis zu rund $20 \%$ Prozent in der Bergzone 2-4. Hier ist erklärend beizufügen, daß diese Direktzahlungen aus verschiedenen Einzelabgeltungen für umweltrelevante Leistungen bestehen. Jede hat ihr Ziel. Die Landwirte sind sich dessen bewußt und reagieren auch gezielt auf die einzelnen Direktzahlungsarten. So ist auch der relativ hohe Anteil in der Bergzone 2-4 ökonomisch unproblematisch und argumentativ folgendermaßen zu rechtfertigen:

- Durch die Gatt-bedingten geringeren Exportbeiträge für Zucht- und Nutzvieh sind vor allem die traditionellen Viehexportregionen im Berggebiet betroffen. Die Preise dürften tatsächlich sinken, jedoch soll ein gleichwertiger Ausgleich über Direktzahlungen erfolgen.

- Von den Kürzungen der Exportbeiträge für Käse sind vor allem die intensiven Milchproduktionsbetriebe im Voralpengebiet betroffen. Aber auch hier ist vorgesehen, durch Direktzahlungen die bereits eingeleiteten Milchpreissenkungen zu einem groBen Teil auszugleichen.

\section{Agrarpolitik 2002}

Und schließlich wird zurzeit das ganze Land wirtschaftsgesetz neu geschrieben. Diese neue "Agrarpolitik 2002» sieht vor, daß im Rahmen des im Gatt vereinbarten AuBenhandelsschutzes im Inland Marktpreise herrschen sollen. Das Gesetz spricht von Richtpreisen, die angestrebt werden. Allerdings sind "Rettungsmaßnahmen» vorgesehen, mittels deren gesorgt wird, daß diese Richtpreise mehr oder weniger erreicht werden. Um verbleibende Überschüsse abzusetzen, sind bei der Milch Zulagen auf verkäster Milch vorgesehen, bei Raps und Zucker Beiträge an die Fabriken für deren gemeinwirtschaftliche Leistungen. Der Staat wird aber keine direkten Markteingriffe mehr wie bisher vornehmen. Daher werden Preise je nach interner Marktlage gelegentlich auch stark fallen bzw. steigen. Die Preise sollen also Angebot und Nachfrage steuern. Nun stellt sich die Frage, ob aus der Sicht der relativ heterogenen Agrarregionen der Schweiz damit Regionalprobleme verbunden sind und ob man gar an regionalpolitische Maßnahmen zu denken hätte.
Mit der neuen Agrarpolitik fallen fast alle staatlichen und halbstaatlichen Eingriffe auf den Märkten weg. An deren Stelle tritt Marktfreiheit. Mit anderen Worten, jeder Landwirt soll das machen, was er für sich für am günstigsten hält. Zwei Elemente werden dadurch neu sehr wichtig, nämlich die Lage seines betrieblichen Standorts einerseits und das Absatzpotential für seine Produkte anderseits. Beide Elemente sind für jeden einzelnen Landwirt grundsätzlich verschieden. Somit läßt sich auch nur noch theoretisch weiter argumentieren, was mögliche Auswirkungen auf einzelne Regionen sein werden. In gewissen Regionen wird die Belieferung von regionalen Märkten das Beste sein; an anderen Orten ist die Produktsammlung durch regionale Genossenschaften, die die Güter auf größere Märkte bringen, das Angebrachte. Touristenregionen mögen saisonale Absatzmärkte haben; andere Regionen haben andere spezifische Marktund Produktionsvorteile.

\section{Zur Nachhaltigkeit der Landwirtschaft unter regionalen Aspekten}

Die neue Agrarpolitik 2002 soll optimale Rahmenbedingungen festlegen, durch die eine private Landwirtschaft auch die öffentlichen Ziele der Gesellschaft erfüllt. Dazu sind die oben beschriebenen Maßnahmen nötig. Diese Maßnahmen haben effizient, effektiv und auch sozialverträglich zu sein. Darauf sei hier nicht weiter eingetreten, bzw. diese werden für die folgenden abschließenden Ausführungen vorausgesetzt. Was uns hier vielmehr beschäftigt, ist die Frage, ob man die beschriebenen neuen agrarpolitischen Konzepte auch mit den regionalen Erfordernissen des Nachhaltigkeitskonzepts (nach Rio 92) in Übereinstimmung bringen kann. Was muß sich diesbezüglich gegenüber der Vergangenheit konkret ändern? Kann eine Bergland wirtschaft überhaupt am Nachhaltigkeitskonzept gemessen werden?

\section{Zum Konzept der Nachhaltigkeit}

Das Konzept der Nachhaltigkeit enthält bekanntlich eine

- ökologische,

- ökonomische (volkswirtschaftliche) und

- soziale Dimension.

Das Konzept verlangt, daß gleichzeitig alle drei Dimensionen minimale Standards aufzuweisen haben oder daß Verbesserungen der einen Dimension nicht auf Kosten einer anderen erfolgen (sog. Win-Win Konzept der Weltbank; SERAGELDIN 1995). Die alte Agrarpolitik hat ganz eindeutig sowohl die ökologische wie auch die volkswirtschaftliche Komponente vernachlässigt und ihr Schwergewicht auf eine sozial motivierte Erhaltungspolitik gelegt. Diese Politik hat zweifellos zu regionalen Strukturen geführt, die im positiven Sinne ländliche Kulturlandschaften prägten. Das Konzept war aber nicht nachhaltig, was die oben erwähnten Probleme belegen. Somit stellt sich zukunftsorientiert die Frage, ob die neue Agrarpolitik die volkswirtschaftliche und die ökologische Dimension verbessert, ohne die soziale zu verschlechtern. 
Darauf wollen wir zuerst eine allgemeine und dann eine regionenbezogene Antwort geben.

\section{Zur Nachhaltigkeit der neuen Agrarpolitik}

Im allgemeinen kann gesagt werden, daß durch den Umbau der Agrarpolitik (inkl. des Gewässerschutzgesetzes) bei allen drei Dimensionen Verbesserungen oder zumindest keine Verschlechterungen entstehen. Die ökologischen Verhältnisse werden verbessert, indem durch Produktpreissenkungen die ökonomischen Anreize reduziert werden, zu intensiv zu produzieren. Das Gewässerschutzgesetz und die spezifischen Beiträge nach Artikel $31 \mathrm{~b}$ des Landwirtschaftsgesetzes wirken in die gleiche Richtung. Ökonomisch bzw. volkswirtschaftlich wird sich die Situation verbessern, weil nach und nach die Marktkräfte zu effizienteren Produktionsstrukturen führen werden. Die soziale Dimension wird auf gezielte Weise über die vorgesehenen Direktzahlungen angestrebt, indem diese wie ein soziales Auffangnetz wirken, ohne den Markt zu stören.

Die regionsbezogene Antwort zur Nachhaltigkeit der anzustrebenden Agrarstrukturen geben wir, indem wir auf die Aktionsparameter zurückgreifen, die der Staat auch unter der neuen Agrarpolitik in seiner Kompetenz behält. Dazu soll Abbildung 2 behilflich sein. Diese bringt zu Ausdruck, daß der Strukturwandel von vier Determinanten abhängt, nämlich den Produktpreisen, den regionalen Arbeitsmarktverhältnissen, den Direktzahlungen und den Strukturanpassungsmaßnahmen. Die Produktpreise sind, wie oben dargelegt, nicht mehr im Kompetenzbereich des Staates. Die regionalen Arbeitsmarktbedingungen, die von Region zu Region sehr unterschiedlich sein können, liegen auch nicht im Einflußbereich der Agrarpolitik, allenfalls der regionalen Wirtschaftspolitik. Dem Staat verbleiben die zwei Aktionsbereiche Direktzahlungen und Strukturmaßnahmen. Mit diesen beiden Maßnahmen hat der Staat die Möglichkeit, regional unterschiedlich zu wirken. Er kann diese nach ungleichen Kriterien anwenden und so auf Besonderheiten unterschiedlicher Agrarstrukturen in einzelnen Regionen

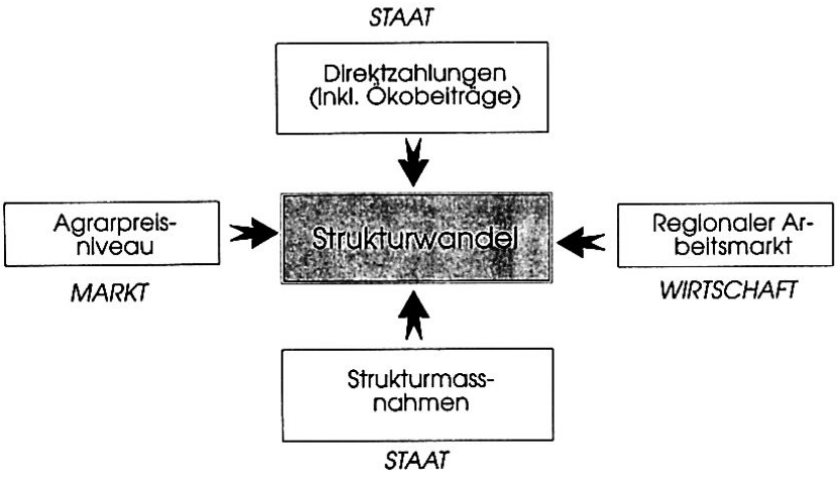

Abb. 2 Determinanten des Strukturwandels.

Rücksicht nehmen. Durch geschickte Kombination dieser zwei Maßnahmengruppen kann er wesentlich beitragen, daß sich die Forderungen nach Nachhaltigkeit effizienter und auch effektiver als bisher erreichen lassen. So wird es grundsätzlich möglich, gleichzeitig sowohl ökologische wie auch ökonomische Forderungen zu erfüllen. Im Sinne eines Leitbildes sprechen wir dann von einer ökologischen Wettbewerbslandwirtschaft.

\section{Literatur}

RIEDER, P., RÖSTI, A., JÖRIN, R. (1994): Auswirkungen der Gatt-Uruguay-Runde auf die schweizerische Landwirtschaft, Inst. für Agrarwirtschaft, ETH Zürich.

SERAGELDIN, I. (1995): Sustainability and the Wealth of Nations: First Steps in an Ongoing Journey, Weltbank, Washington.

BAUR, P. (1995): Ökologische Direktzahlungen. Ein Diskussionsbeitrag aus ökonomischer Sicht. In: Agrarwirtschaft und Agrarsoziologie, Schweiz. Gesellschaft für Agrarwirtschaft und Agrarsoziologie, Nr. 2. 